\title{
Intelligent Seamless Vertical Handoff Algorithm for the next generation wireless networks
}

\author{
Jiping LV \\ Hitachi (China) Research \& \\ Development Corporation \\ Yuanchen $\mathrm{Ma}$ \\ Hitachi (China) Research \& \\ Development Corporation \\ Satoshi Yoshizawa \\ Hitachi (China) Research \& \\ Development Corporation \\ 301, North Tower C, Raycom infotech 301, North Tower C, Raycom infotech 301, North Tower C, Raycom infotech \\ Park, 2 kexueyuan Nanlu, Hai Dian Park, 2 kexueyuan Nanlu, Hai Dian \\ District, Beijing, 10080, China \\ District, Beijing, 100080 China \\ Park, 2 kexueyuan Nanlu, Hai Dian \\ District, Beijing, 100080, China \\ jplv@hitachi.cn \\ ycma@hitachi.cn \\ syoshizawa@hitachi.cn
}

\begin{abstract}
With the rapid development of wide variety of wireless technologies, it is envisioned that the future network will be a convergence of various wireless access technologies providing users with the best anywhere, anytime connections to the Internet. In such environment, it is a critical problem to enable a mobile node to intelligently select wireless access networks and achieve seamless handoff performance. This paper proposes a vertical handoff algorithm which enables a mobile node to intelligently select wireless access network among multiple access technologies, synthetically considering network characteristics and status, such as access bandwidth, response time, data loss, network congestion status, etc, along with access cost and received signal strength (RSS). With the proposed algorithm, wireless network utilization can be optimized according to user's strategy and network conditions, and real-time applications can be maintained without packet loss during handoff process. A prototype system is implemented and experimental results are analyzed.
\end{abstract}

\section{Categories and Subject Descriptors}

C.2.2 [Network Protocols]: Protocol Architecture; C.2.1 [Computer-Communication Networks]: Network Architecture and Design - wireless communication

\section{General Terms}

Algorithms, Design, Experimentation

\section{Keywords}

Vertical handoff, handoff algorithm, intelligent handoff

\section{INTRODUCTION}

With the rapid growth in demand for various wireless communication services, many wireless communication

Permission to make digital or hard copies of all or part of this work for personal or classroom use is granted without fee provided that copies are not made or distributed for profit or commercial advantage and that copies bear this notice and the full citation on the first page. To copy otherwise, or republish, to post on servers or to redistribute to lists, requires prior specific permission and/or a fee.

MOBILWARE 2008, February 13-15, Innsbruck, Austria

Copyright @ 2008 ICST 978-1-59593-984-5

DOI 10.4108/ICST.MOBILWARE2008.2553 technologies have emerged, such as GPRS (General Packet Radio Service), UMTS (Universal Mobile Telecommunication System), CDMA (IS-95 A/B and CMDA2000) and WiMAX (Worldwide Interoperability for Microwave Access), WLAN (Wireless local area networks), etc. To improve the system resource utilization and realize interworking of different wireless access technologies, the future wireless network is envisioned as a convergence of various wireless access technologies [11], enabling users to be always best connected to the Internet and continue using their applications without transmission disruption while they are moving between different wireless access technologies. In such environment, it is a critical issue for mobile nodes to intelligently select access network and maintain active connections while they are roaming between different wireless access technologies.

This paper proposes a universal vertical handoff algorithm which enables mobile nodes to intelligently select wireless access network, considering not only RSS, but also access cost, network characteristic and status, such as access bandwidth, response time from MN (Mobile Node) to correspondent nodes, data loss, number of hops from the MN to correspondent nodes, network congestion, etc. In the algorithm, an equation is proposed, and based on the calculation result with the equation, MN can easily select access network among multiple access technologies according to user's strategy and network performance. Also the equation applies to all kinds of handoff scenarios, such as handoff from global coverage network to local coverage network and vice versa, handoff between local coverage networks, and handoff between global coverage networks.

Several vertical handoff algorithms have been proposed. In [1], a vertical handoff scheme named VHOST (Vertical Hand-off through Seamless TCP-migration) is proposed. The handoff scheme applies TCP-migration approach to vertical handoff system to migrate connections from old communication system to the new one. Although additional functions such as foreign agent and home agent are not needed, it has to modify DNS and all the high-layer protocols and applications accordingly, thus modifications in all the terminals are necessary. For handoff decision, VHOST makes vertical handoff decision based on the RSS, the characteristics of bandwidth, and coverage area. It does not take other factors into consideration, such as access cost, response time, hops from $\mathrm{MN}$ to correspondent nodes, network congestion status, data loss, etc. In [2], authors propose a vertical handoff algorithm for handoff between WLAN and CDMA. The 
algorithm makes handoff decision based on RSS and velocity. Though velocity is helpful for handoff decision, system resources will be consumed to get MN's velocity. In [3], authors propose integration of third-generation wireless networks with local-area 802.11 wireless technologies. Two integration approaches were presented, tight integration approach and loose integration approach, and the latter is advocated as the preferred approach. In the implementation of loose integration, vertical handoff decision is made based on the RSS, RSS threshold, and interface priority. In [5], a vertical handoff decision algorithm, Self-Adaptive Vertical handoff Algorithm (SAVA), is proposed. SAVA makes handoff decision considering long term movement region and short term movement trend of mobile hosts. [4] presents an interworking architecture between 3PGG and WLAN system, [6] proposes an end-to-end multi-path transmission scheme for stream media, [7] presents details of design and implementation of WLAN/CDMA2000 interworking architecture. We can see, till now, there is no proposed vertical handoff algorithm which makes handoff decision with such a universal equation, synthetically takes network characteristics and performance, access cost, and RSS into considerations.

The rest of the paper is organized as follows. Section II describes the proposed vertical handoff algorithm in detail. Section III describes the vertical handoff system design and implementation. In Section IV, experimental environment is described and experimental results are presented to investigate the performance of the proposed algorithm. Conclusion and future work is given in Section V.

\section{PROPOSED INTELLIGENT SEAMLESS VERTICAL HANDOFF ALGORITHM}

In the future convergence network which integrates multiple wireless access technologies, the most important and critical issue is how to select wireless access network and when to trigger handoff operation to achieve seamless handoff performance.

To solve this critical problem, we propose the following equation to evaluate the total performance of different access technologies.

$$
V_{i}=S\left(\text { Cost }_{\min } / \operatorname{Cost} \times w_{c}+Q \times w_{q}\right)
$$

MN makes handoff decision according to the total network performance comparison result, and can trigger handoff operation before it loses its connection to the previous access network.

$\boldsymbol{V}_{\boldsymbol{i}}$ denotes interface i access network's total performance, MN makes handoff decision according to the value of $\boldsymbol{V}_{\boldsymbol{i}}$ and selects the access network with the largest value of $\boldsymbol{V}_{\boldsymbol{i}}$.

$\boldsymbol{S}$ denotes RSS Let $\mathbf{H}$ denote high threshold of RSS, and $\mathbf{L}$ denote low threshold of RSS. The value of $\boldsymbol{S}$ is defined as the following equation (2).

$$
s= \begin{cases}1 & \text { RSS }>\text { H } \\ \text { RSS } / H & \mathrm{~L} \leq \mathrm{RSS} \leq \mathrm{H} \\ 0 & \mathrm{RSS}<\mathrm{L}\end{cases}
$$

When RSS is larger than its high threshold, the value of $\boldsymbol{S}$ is set to 1 , and when RSS is lower than its low threshold, the value of $\boldsymbol{S}$ is set to 0 . This makes $\mathrm{MN}$ immediately handoff to other network when the current network's RSS is lower than its low threshold, and needs not to consider other factors. On the other hand, when RSS is larger than its high threshold, MN only needs to consider other factors, such as access cost, network performance, and selects cheaper access network with better performance. As the RSS decreases, the total performance value of $\boldsymbol{V}_{\boldsymbol{i}}$ will decreases at fast speed also. Before RSS becomes lower than its low threshold, the value of $\boldsymbol{V}_{\boldsymbol{i}}$ in current network will be lower than that in other network, and MN will trigger handoff operation. In this way, MN can set up the new link before it loses its previous link, and achieves seamless vertical handoff performance.

Cost denotes expense users pay for access network provider. It enables MN to select the cheapest access network when other network factors are equal.

Cost $_{\text {min }}$ denotes the minimum cost of all access networks. It is used here to realize normalized unit, i.e. Cost $_{\text {min }} / \operatorname{Cost}_{\in}[0,1]$.

$\boldsymbol{w}_{\boldsymbol{c}}$ denotes the weighing coefficient of Cost.

$\boldsymbol{w}_{\boldsymbol{q}}$ denotes the weighing coefficient of network quality of service $Q$.

$$
\text { Here } \boldsymbol{w}_{\boldsymbol{c}}, \boldsymbol{w}_{\boldsymbol{q}} \in[0,1] \text { and } \boldsymbol{w}_{\boldsymbol{c}}+\boldsymbol{w}_{\boldsymbol{q}}=1 \text {. }
$$

$\boldsymbol{Q}$ denotes network performance, where

$$
\boldsymbol{Q}=\sum_{i=1}^{n} \mathrm{Xi} \times W i, i=\{1,2,3,4 \ldots\}
$$

$\boldsymbol{X i}$ denotes factor which influences network performance, each $\boldsymbol{X i}$ representing one factor, such as access bandwidth, network congestion status, response time from MN to correspondent nodes, data loss, hops from MN to correspondent nodes, etc. We do not recommend all factors to be used to evaluate network performance at the same time, but suggest users select main factors which can reflect network performance according to their service requirements and network deployment status.

$\boldsymbol{W i}$ denotes the weighing coefficient of $\boldsymbol{X i}$, where

$$
\sum_{i=1}^{n} W i=1, i=\{1,2,3, \ldots\}
$$

If user does not want to consider factor $\boldsymbol{X i}$ which has been defined and implemented, just set the value of $\boldsymbol{W i}$ to 0 .

The equation applies to all kinds of handoff scenarios, and makes it easy for mobile node to make handoff decision and select best access network synthetically taking RSS, access cost and network performance into consideration. It enables $\mathrm{MN}$ to trigger handoff operation before it loses its previous link, so that mobile node can continue real-time applications without packet loss during the handoff process.

In the first step of our prototype, for the network performance $\boldsymbol{Q}$, only the bandwidth factor is considered, and other factors will be considered in the future implementation. 


\section{VERTICAL HANDOFF SYSTEM DESIGN AND IMPLEMENTATION}

To make MN intelligently select access technology, the previous equation will be used to compare different access network's performance. When $\mathrm{MN}$ is located or moving in the area which is covered with multiple wireless access technologies, it will evaluate and compare different access networks' total performance. If other access technology's total performance is better than the current one, MN will handoff to the better access technology. During the handoff process, to reduce handoff delay and packet loss, mobile IP [8-9] is also used to achieve seamless handoff performance. Mobile IP permits MN to continue using its permanent home address as it moves around the Internet, making the movement of $\mathrm{MN}$ transparent to high layer protocols and applications. Thus applications will not be interrupted during handoff process.

Figure 1 shows the software architecture of MN. Five modules are added compared to normal terminal, Virtual Driver, Mobile IP, Network Performance Computation, Handoff Decision and GUI for Handoff. Mobile IP module includes mobile IPv4 and Mobile IPv6. The proposed software architecture does not require any modification in high-layer protocols nor applications, while providing intelligent seamless vertical handoff.

Network Performance Computation module computes different access networks' performance according to our proposed equation (1) in section II. In the first step of our implementation, for network performance $\boldsymbol{Q}$, only the bandwidth factor is considered, other factors such as response time from $\mathrm{MN}$ to correspondent nodes, data loss, congestion status, etc, will be considered in the future implementation. So the equation to get the value of total network performance for our first prototype is as followed,

$$
\begin{aligned}
& V_{i}=S\left(\operatorname{Cost}_{\min } / \operatorname{Cost} \times w_{c}+B \times w_{b}\right) \\
& w_{c}+w_{b}=1, \\
& w_{c}, w_{b}, \text { Cost }_{\min } / \text { Cost, } B \in[0,1] \text {, } \\
& \boldsymbol{S}= \begin{cases}1 & \text { RSS }>\text { H } \\
\text { RSS } / H & \text { L } \leq \text { RSS } \leq H \\
0 & \text { RSS }<\text { L }\end{cases}
\end{aligned}
$$

$\boldsymbol{B}$ denotes the normalized bandwidth, where

$$
B=\text { Band } / B_{\text {max }}
$$

Band denotes the access bandwidth of interface $\boldsymbol{i}$.

$\boldsymbol{B}_{\max }$ denotes the maximum access bandwidth of all access technologies.

$\boldsymbol{w}_{\boldsymbol{b}}$ denotes the weighing coefficient of $\boldsymbol{B}$.

$\boldsymbol{V}_{i}, \boldsymbol{S}$, Cost $_{\min }$, Cost, $\boldsymbol{w}_{\boldsymbol{c}}$, RSS, $\mathbf{H}$ and $\mathbf{L}$ denote the same meaning as defined in section II.

The value of $\boldsymbol{S}$, Cost $_{\min }$, Cost, $\boldsymbol{w}_{\boldsymbol{c}}, \mathbf{R S S}, \mathbf{H}$ and $\mathbf{L}$ is predefined, and can be viewed and modified in the GUI for Handoff.

Handoff Decision module selects access network with the best network performance at startup and makes handoff decision during moving process. It receives RSS from virtual driver module and gets variable value such as access cost and weighing coefficient from GUI for Handoff module, then call Network Performance Computation module to compute detected access network's total performance according to equation (5). If other access network's total performance is better than the current one, the module will trigger mobile IP module to send registration request message to $\mathrm{HA}$, and notify the virtual driver module to send mobile IP signal message through the new network interface. After it receives the notification that mobile IP registration process has successfully finished, it will notify virtual driver to transfer the data traffic to the new network interface.

Mobile IP module enables MN continue using its permanent home address as it moves around the Internet, enabling continuity of sessions. To support IPv4 and IPv6, both mobile IPv4 [8] and mobile IPv6 [9-10] are implemented. In mobile IPv4, there is foreign agent (FA) function compared to mobile IPv6. FA helps MN finishing new position registration process. When MN moves from one network to another, it will get a new IP address with the new networks' prefix, called Care of Address (CoA). Then MN sends binding registration request message to its home agent (HA) to update its CoA. After $\mathrm{MN}$ receives successful binding response message from HA, it will notify Handoff Decision module that mobile IP registration procedure has finished. Then Handoff Decision module will notify virtual driver to use the new network interface for data traffic.

Virtual Driver module manages multiple network interfaces. It collects RSS from different network interfaces and reports the information to Handoff Decision module. It also decides which interface is used for data transmission and which interface is used for signal message.

GUI for Handoff module provides user with network information, such as the available link and RSS. It provides interface for user to input and modify the variable value of different access technologies such as access cost and its weighing coefficient, etc.

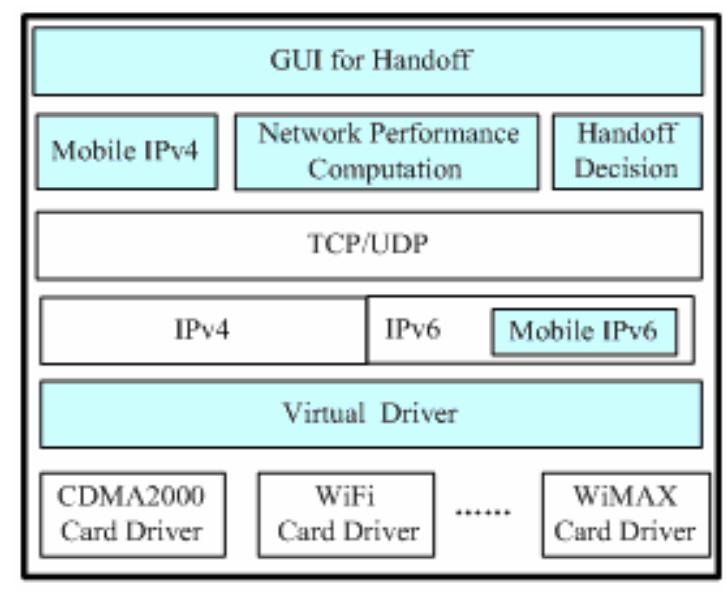

Figure 1. Architecture of MN.

\section{PERFORMANCE EVALUATION}

\section{A. Experimental Environment}

To verify the feasibility of the proposed intelligent seamless vertical handoff algorithm, we have implemented a prototype 
system of vertical handoff between CDMA2000 1xEV-DO and WLAN.

Figure 2 shows the experimental environment. The system includes CDMA2000 1xEV-DO access network, WLAN access network and Home network. AAA, HA are deployed in the home network. PDSN (Packet Data Serving Node) works as the foreign agent in 1xEV-DO system, and PDIF (Packet Data Interworking Function) is the foreign agent in WLAN system. There are two clients in the system, one is a mobile node which is equipped with two network cards, 1xEV-DO card and WiFi card, the other is a common CN (Correspondent Node). Both clients run Video Telephone (VT) application to verify whether the vertical handoff system is able to provide seamless handoff service for real-time application. A special device is added to control the RSS of AP in WLAN.

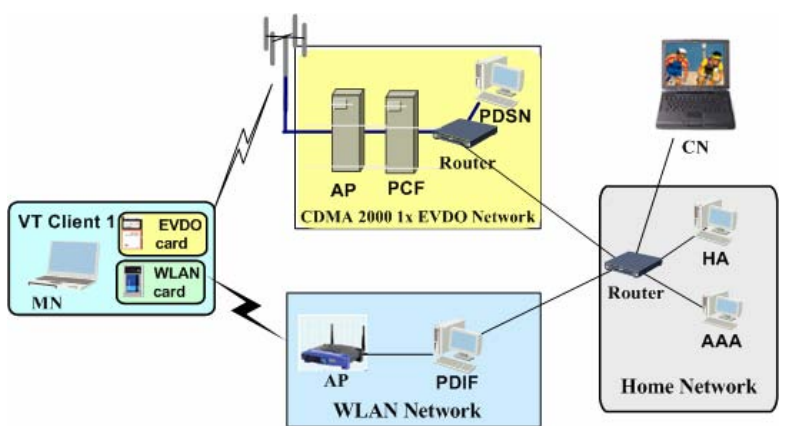

Figure 2. Experimental environment of vertical handoff between 1XEV-DO and WLAN.

\section{B. Performance Evaluation}

To verify the proposed vertical handoff algorithm, we have designed and performed several experiments.

\section{Experiment 1}

In the first experiment, we aim to verify whether $\mathrm{MN}$ can intelligently select access network and the system can support real-time application.

We set the variable value in equation (5) for experiment 1 as table 1.

Table 1. Variable value for experiment 1

\begin{tabular}{|c|c|c|c|c|c|c|}
\hline & Band & Cost & $B_{\max }$ & Cost $_{\min }$ & $w_{\boldsymbol{c}}$ & $\boldsymbol{w}_{\boldsymbol{b}}$ \\
\hline WLAN & 11 & 0.2 & \multirow{2}{*}{11} & 0.2 & 0.5 & 0.5 \\
\cline { 1 - 5 } 1xEV-DO & 2.45 & 0.6 & & & & \\
\hline
\end{tabular}

First RSS in WLAN and 1xEV-DO are set to above their high thresholds. Initially, we observe that MN selects WLAN network automatically. From table 1 we can see that WLAN system provides wider access bandwidth with cheaper cost compared to $1 \mathrm{xEV}$-DO system, so the total performance value $\boldsymbol{V}_{\boldsymbol{i}}$ of WLAN is larger than that of $1 \mathrm{xEV}-\mathrm{DO}$, and $\mathrm{MN}$ will select WLAN. Then VT session will be set up between MN and CN. Then the RSS of WLAN is gradually tuned down, after some time the MN handoffs to $1 \mathrm{xEV}$-DO system. During the handoff process, the VT session is not interrupted. In the equation (5), we can see that as the RSS lowers down, its total performance value Vi lowers down also, before the RSS in WLAN lowers to its low threshold,
$\mathrm{Vi}$ in WLAN will be lower than that in $1 \mathrm{xEV}-\mathrm{DO}$, so MN will trigger handoff operation before it loses its previous link. In this way handoff is seamless. Then the RSS of WLAN is tuned up gradually, the MN handoffs to $1 \mathrm{xEV}-\mathrm{DO}$ system again. Video session does not be interrupted during handoff process either.

Figure 3 shows the captured packets. The horizontal axis shows the packet number sent from $\mathrm{MN}$ to $\mathrm{CN}$, the vertical axis shows the transmission time from $\mathrm{MN}$ to $\mathrm{CN}$. The left part and right part show MN access through WLAN, the middle part shows MN access through $1 \mathrm{xEV}-\mathrm{DO}$. As the WLAN provides higher bandwidth, the transmission time in WLAN is lower than that in $1 \mathrm{xEV}$-DO system. When we tune down RSS in WLAN, MN handoffs from WLAN to $1 \mathrm{xEV}-\mathrm{DO}$, and transmission time is larger than that in WLAN, but there is no packet loss in the handoff process as shown in the figure. When the RSS in WLAN is larger, MN handoffs back to WLAN, and transmission time is lower than that in $1 \mathrm{xEV}-\mathrm{DO}$, and there is no packet lost in the handoff process either.

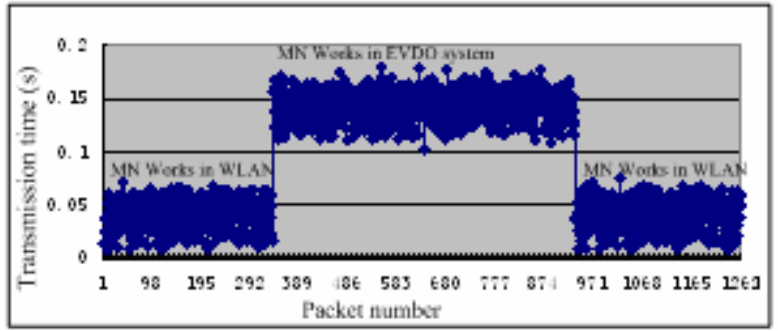

Figure 3. Transmission time from $\mathrm{MN}$ to $\mathrm{CN}$ as RSS changed

Experiment 1 proves that $\mathrm{MN}$ can intelligently select access network and the handoff delay is minimized to satisfy real-time application and there is no packet lost during handoff process.

\section{Experiment 2}

This experiment aims to test whether $\mathrm{MN}$ can select access network according to our strategy. The experiment environment is same as in experiment 1 . Set the initial value same as in table 1. RSS of 1xEV-DO and WLAN is larger than the high threshold. VT session is set up between MN and CN. Initially MN connects to WLAN system. Then we modify the network parameter via GUI, increasing the value of Cost and its weighing coefficient $\boldsymbol{w}_{c}$ in WLAN as in table 2, then MN handoffs to $1 \mathrm{xEV}$-DO system. Figure 4 showed the experimental result. After cost and its weighing coefficient in WLAN are increased, MN handoffs to the cheaper access network as we expected.

Table 2. Variable value for experiment 2

\begin{tabular}{|c|c|c|c|c|c|c|}
\hline & Band & Cost & $\boldsymbol{B}_{\max }$ & Cost $_{\min }$ & $\boldsymbol{w}_{\boldsymbol{c}}$ & $\boldsymbol{w}_{\boldsymbol{b}}$ \\
\cline { 1 - 6 } WLAN & 11 & 1.2 & \multirow{2}{*}{11} & 0.6 & 0.9 & 0.1 \\
\cline { 1 - 5 } 1xEV-DO & 2.45 & 0.6 & & & & \\
\hline
\end{tabular}




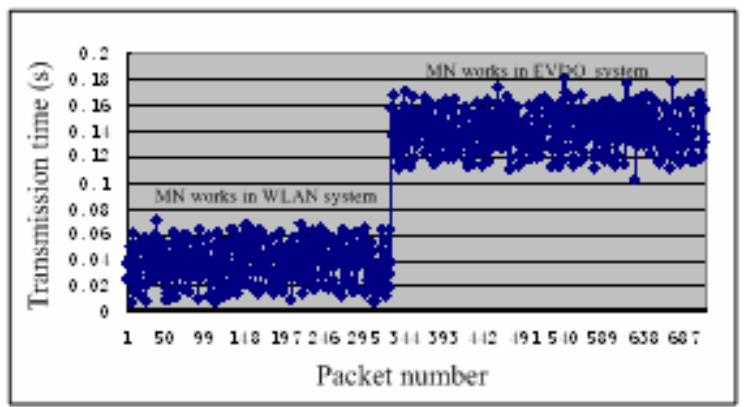

Figure 4. Transmission time from $\mathrm{MN}$ to $\mathrm{CN}$ as cost changed

This experiment proves that $\mathrm{MN}$ will select the cheaper access network when other parameters do not change.

\section{CONCLUSION AND FUTURE WORK}

In this paper, an intelligent seamless vertical handoff algorithm is proposed which enables mobile nodes to select access network intelligently, synthetically considering RSS, access cost, network performance, such as bandwidth, data loss, response time from MN to correspondent nodes, etc, and the proposed handoff equation applies to all kinds of handoff scenarios. In this paper, a prototype system of vertical handoff between CDMA2000 1xEVDO and WLAN is implemented. In this prototype system, bandwidth is considered as the network performance factor. Other factors such as response time, data loss, etc will be considered in the future. The prototype system proves that our proposed vertical handoff algorithm enables $\mathrm{MN}$ to select access network intelligently according to access network status, and there is no packet loss or transmission break for real-time application during handoff process.

Our final target is to realize a convergence system integrating wide variety of wireless access technologies. In the next step, we will implement a prototype which permits mobile nodes to intelligently select access network and seamlessly handoff among multiple wireless access technologies, including $1 x E V-D O$, WLAN, WiMAX and UMTS, where mobile nodes make handoff decision not only based on RSS, access cost, access bandwidth, but also based on response time, data loss, congestion status, etc.

\section{REFERENCES}

[1] Rong-Jyh Kang; Hsung-Pin Chang; Ruei-Chuan Chang, "A Seamless Vertical Handoff Scheme", Wireless Internet, 2005.
Proceedings. First International Conference on 10-14 July 2005 Page(s):64 - 71.

[2] Kyung-Soo Jang, Jang-Sub Kim, Ho-Jin Shin, Dong-Ryeol Shin, "A novel vertical handoff strategy for integrated IEEE 802.11 WLAN/CDMA networks”, Computer and Information Science, 2005. Fourth Annual ACIS International Conference on 2005 Page(s):616 - 621.

[3] Buddhikot, M., Chandranmenon, G., Han, S., Lee, Y.W., Miller, S., Salgarelli, L., "Integration of 802.11 and thirdgeneration wireless data networks”, INFOCOM 2003. Twenty-Second Annual Joint Conference of the IEEE Computer and Communications Societies. IEEE Volume 1, 30 March-3 April 2003 Page(s):503 - 512 vol.1.

[4] Ahmavaara, K., Haverinen, H., Pichna, R., "Interworking architecture between 3GPP and WLAN systems", Communications Magazine, IEEE Volume 41, Issue 11, Nov. 2003 Page(s):74 - 81.

[5] Liu Min, Li Zhongcheng, Xiao-Bing Guo, Eryk Dutkiewicz, Ming-Hui Wang, "SAVA: A Novel Self-Adaptive Vertical Handoff Algorithm for Heterogeneous Wireless Networks”, IEEE Global Communications Conference 2006 (GLOBECOM 2006), San Francisco, USA, November 27 December 1, 2006.

[6] Yi Pan, Meejeong Lee, Jaime Bae Kim, Tatsuya Suda, “An end-to-end multi-path smooth handoff scheme for stream media”, WMASH 2003: 64-74.

[7] Buddhikot, M., Chandranmenon, G., Seungjae Han, YuiWah Lee, Miller, S., Salgarelli, L., "Design and implementation of a WLAN/cdma2000 interworking architecture”, Communications Magazine, IEEE, Volume 41, Issue 11, Nov. 2003 Page(s):90 - 100.

[8] C. Perkins, Ed, “IP Mobility Support for IPv4”, RFC 3344, IETF, August 2002.

[9] D. Johnson, C. Perkins, J. Arkko, "Mobility support in IPv6”, RFC 3775, IETF, June 2004.

[10] LV Ji-ping, XU Ming-wei, WU Qian, DENG Hui, “A Survey of Research on Fast Handoff for Mobile IPv6", Journal of Chinese Computer Systems, Vol. 28, No.7, Jul. 2007, pp. 1153-1161.

[11] A.H. Zahran and B. Liang, "Performance evaluation framework for vertical handoff algorithms in heterogeneous networks", Proc ICC 2005, May 2005, pp. 173-178. 